\title{
A note on the within-cell layout problem based on operation sequences
}

Article in Production Planning and Control · January 1996

DOI: $10.1080 / 09537289608930330$

CITATIONS

11

1 author:

\section{Selim Akturk}

Bilkent University

74 PUBLICATIONS $\quad \mathbf{1 , 4 1 0}$ CITATIONS

SEE PROFILE
READS

67

Some of the authors of this publication are also working on these related projects: 


\title{
A note on the within-cell layout problem based on operation sequences
}

\author{
M. SELIM AKTURK
}

Keywords cellular manufacturing, cell layout, operation sequence, part similarity

\begin{abstract}
The existing studies in the literature usually ignore the within-cell layout problem while forming part families and manufacturing cells. A new approach is proposed to solve the part-family and machine-cell formation problem and to determine the spatial arrangement of machines in each cell simultaneously. Furthermore, a dissimilarity measure between parts based on operation sequences is presented since the operation sequences not only specify the type of machine tools needed but also impact the flow of material. As a result, we can both minimize the material handling cost and streamline the material flow in each cell.
\end{abstract}

\section{Introduction}

In a recent article, Kamrani and Parsaei (1994) proposed a methodology which used design and manufacturing attributes simultaneously to form manufacturing cells. Their methodology was implemented in two phases. Phase I of the methodology identified part families using a coding system based on the dissimilarity measures between parts. Phase II of the methodology determined the number of cells, assignment of the parts to the cells and the number of machine types, required to meet the production demand and other constraints, based on relevant operational costs, including a material handling cost. In phase I, they proposed a coding system, called KAMKODE, whereas in phase II they developed a mixed-integer mathematical model to determine the manufacturing cell formation.

Their mathematical formulation related with the material handling cost is given below, where other objective function terms and constraints are dropped for clarity.

Index sets

$$
\begin{array}{ll}
p & : \text { part }, p=1,2, \ldots, P \\
c & : \text { cell, } c=1,2, \ldots, C
\end{array}
$$

Decision variable

$$
\begin{aligned}
& Y_{p c}: 0-1 \text { binary variable which is equal to } 1 \text { if part } \\
& \quad p \text { is assigned to cell } c
\end{aligned}
$$

Coefficients

$\mathcal{N M} V_{p}$ : number of the moves for part $p$

$C H_{p c}$ : average material handling cost of part $p$ in cell $c$

$d_{p} \quad$ : total quantity of part $p$ demanded annually

$B M H$ : annual budget available for material handling of all parts in all cells

Mathematical model

$$
\begin{gathered}
\min \sum_{p=1}^{P} \sum_{c=1}^{C}\left(\mathcal{N} M V_{p}-1\right) d_{p} C H_{\mathrm{pc}} \mathrm{Y}_{\mathrm{pc}} \\
\text { s.t. } \sum_{p=1}^{P} \sum_{c=1}^{C}\left(\mathcal{N} M V_{p}-1\right) d_{p} C H_{p c} r_{p c} \leq B M H
\end{gathered}
$$

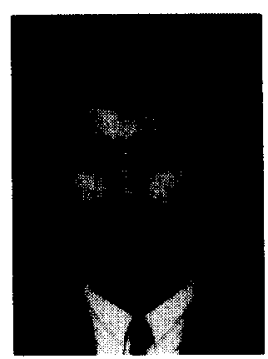

Author: M. Selim Akturk, Department of Industrial Engineering, Bilkent University, 06533 Bilkent, Ankara, Turkey.

M. SELIM AKTURK is assistant professor of industrial engineering at Bilkent University, Turkey. He holds a PhD in industrial engineering from Lehigh University, USA, and BSIE and MSIE from METU, Turkey. His current research interests include hierarchical planning and control of large-scale systems, cellular manufacturing systems, and advanced manufacturing technologies. Dr Akturk is a senior member of IIE, a member of TIMS and Decision Sciences, and full member of ORSA. 
The material handling cost for each part correctly defined as a function of the number of moves for part $p, \mathcal{N M V}$. But, there are erroneous assumptions in this formulation. First of all, $\mathcal{N} M V_{p}$ is taken as a coefficient even though it should be a decision variable. Because the number of moves for a certain part is not fixed, and depends on the spatial arrangement of machines in each cell. Therefore, the within-cell layout problem should be solved first to calculate the number of moves for each part. Moreover, the cell layout problem is a decision variable too since it depends on the parts that are assigned to that cell and their operation sequences. Finally, this formulation does not distinguish the intracell moves from the intercell moves.

The purpose of this paper is to propose an alternative formulation to the mathematical model developed by Kamrani and Parsaei (1994) so that we can solve the part-family and machine cell formation (PFMCF) and within-cell layout problems simultaneously. The remainder of this paper is organized as follows. In the following section, a review of related literature is presented. The proposed mathematical model is discussed in Section 3. An example problem is provided in Section 4 along with a new dissimilarity measure based on operation sequences. Finally, some concluding remarks are given in Section 5.

\section{Background}

Offodile et al. (1994) provide a comprehensive review of the cellular manufacturing $\langle\mathrm{CM})$ literature and present an extensive bibliography of the PFMCF problems by citing more than 100 group technology (GT) related studies. The existing studies often deal with the part-families and they do not make any effort on the spatial arrangement of machines inside cells, even though the cell layout can have a significant impact on the cell loading problem, the amount of work-in-process inventories, throughput, and throughput variability. Greene and Sadowski (1982) discussed that the modified flow shop idea is more appropriate to take advantage of $\mathrm{CM}$ benefits. In a modified flow shop, each part will move in only one direction, i.e. it is not necessarily processed by all of the machines, but backtracking is not allowed. Parts with routeings in the reverse direction with the cell layout are called backtracking parts. The importance of eliminating backtracking parts is also emphasized by Sarker et al. (1994). Modified flow has the advantages of less flow distance, easier material handling and less-complicated scheduling-control problems. Another advantage is the applicability of Kanban systems, since the cell configuration must be flow based in a Kanban system to reduce transport and waiting times, and to highlight interdependencies between the work centres. Moreover, the application of GT cells in advanced manufacturing systems such as JIT and FMS will certainly enhance the design and operational efficiency of such systems. A review by Gunasakeran $e t a l$. (1994) reveal that there is a need to develop GT models and techniques, which will focus on the application to such advanced manufacturing systems. This needs to consider various operational and technological constraints of the manufacturing systems as well as suitable criteria while forming part families and GT cells.

Currently similarity coefficients typically rely on substitute measures, such as common machines required in processing, to evaluate the similarity between parts. Similarity coefficients that employ more direct measures, such as similarities between parts in terms of operation sequences, may yield better results. The operation sequences not only specify the type of machine tools needed but also impact the flow of the material.

There are a few studies in the literature that propose similarity coefficients based on part routeings. Tam (1990) used the Levenshtein's distance measure to represent any part routeing as a string of characters, and to find the similarities between two routeings based on the operations sequences. Choobineh (1988) also presented a similarity measure based on the operation sequences and proposed an integer programming model. Vakharia and Wemmerlöv (1990) proposed a four-stage cell formation algorithm based on the operation sequences to establish a flow-line cell under the restriction that the capital available to make additional investments in equipment is limited and the utilization of cell equipment must be above an acceptable level. They also emphasized the importance of the within-cell layout problem to achieve a flow-line cell by stating its advantages over the job shop cells. Harhalakis et al. (1990) developed a heuristic method of grouping machines in order to minimize the within-cell material movements under the constraint that the specified number of machines per cell was not exceeded. Most of the above methods, however, ignored the interaction between the part-families and the machine-cell formation process, which might affect the structure and the efficiency of the cells as discussed above. Furthermore, the configuration and the performance measures of the cell must be considered throughout the design process in order to provide information for the selection of the best part-family and machine-cell formation in conjunction with the spatial arrangements of machines in each cell.

Table 1. Operation sequences for each part.

\begin{tabular}{|c|c|c|c|c|c|c|}
\hline \multirow{2}{*}{$\frac{\text { Part number }}{1}$} & \multicolumn{6}{|c|}{ Operation sequence } \\
\hline & A & $\mathrm{D}$ & $E$ & $F$ & & \\
\hline 2 & $\mathrm{C}$ & $\mathrm{F}$ & & & & \\
\hline 3 & $\mathrm{~A}$ & B & $\mathrm{D}$ & $\mathrm{E}$ & & \\
\hline 4 & B & $\mathrm{C}$ & $\mathrm{D}$ & $\mathrm{E}$ & $\mathrm{F}$ & \\
\hline 5 & $\mathrm{~A}$ & B & $\mathrm{C}$ & $\mathrm{D}$ & F & $\mathrm{F}$ \\
\hline
\end{tabular}


Table 2. Final part-family machine-cell formation.

\begin{tabular}{cccccccccccccccc}
\hline Cell number & Family number & \multicolumn{1}{c}{ Part family } & \multicolumn{1}{c}{ Machine type } \\
\hline 1 & 1 & 1 & 3 & 2 & & 5 & A & & B & & D & & E & & F \\
2 & 2 & & & 3 & & 4 & & & C & & D & & E & & F \\
\hline
\end{tabular}

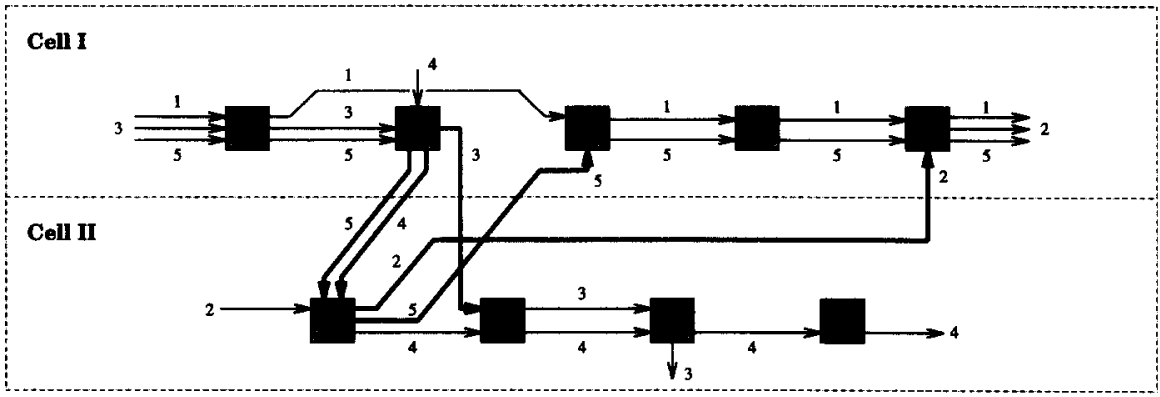

Figure 1. Flow patterns within each cell.

\section{The mathematical model}

The solution of this model will specify the spatial arrangement of machines in each cell that minimizes the total material handling cost and streamlines the material flow in addition to the part-family and machine-cell formation. A part may start production at any machine and may leave the cell after any machine, but backtracking is not allowed due to the modified flow shop assumption. For convenience, it is assumed that parts flow from left to right. Furthermore, exactly one machine can be assigned to each location. If a part moves from location $a$ to location $b$ where $a<b$, the number of skippings is equal to $[(b-a)-1]$. The additional decision variables and parameters are given below, in which subscripts $k$ and $l$ refer to machine types, whereas $M$ is a set of all machine types.

Decision variables

$\gamma_{k l c}: 0-1$ binary variable which is equal to 1 if machine type $k$ is placed before machine type $l$ (not necessarily immediately) in cell $c$

$m_{k c} \quad$ : number in the sequence of machine type $k$ in cell $c$

$\mathcal{N M V} V_{p}:$ total number of skippings for part $p$

Parameters

$x_{p k l}: 0-1$ binary indicator which is equal to 1 if part $p$ should be processed by machine type $k$ immediately before machine type $l$

$U \quad$ : a very large constant

Mathematical model

$$
\min \sum_{p=1}^{P} \sum_{c=1}^{C} \mathcal{N} M V_{p} d_{p} C H_{p c}
$$

Subject to

$$
\begin{aligned}
& \sum_{p=1}^{P} \sum_{c=1}^{C} \mathcal{N} M V_{p} d_{p} C H_{p c} \leq B M H \\
& Y_{p c} x_{p k l}\left(m_{l c}-m_{k c}\right) \geq 1 \quad \forall p, c, k, l \text { (5) } \\
& \left(m_{l c}-m_{k c}\right)+U\left(1-\gamma_{k l c}\right) \geq 1 \quad \forall k, l, c \\
& \left(m_{k c}-m_{l c}\right)+U \gamma_{k l c} \geq 1 \quad \forall k, l, c \\
& \sum_{k \in M} \sum_{l \in M} Y_{p c} \mathrm{x}_{p k l}\left(m_{l c}-m_{k c}-1\right)-\mathcal{N} M V_{p}=0 \quad \forall p, c \\
& Y_{p c}, \gamma_{k l c}=0,1 \text { and } m_{k c}, \mathcal{N M} V_{p} \text { are integer } \forall p, c, k, l \text { (9) }
\end{aligned}
$$

In this formulation, the objective function and constraint 4 are similar to the Kamrani and Parsaei's model, but $\mathcal{N} M V_{p}$ now becomes a decision variable so the $r_{p c}$ can be dropped from the material handling equations. Constraint 5 is an inequality which determines the machines' sequence in a cell by utilizing the immediate precedence data to determine the machine arrangements. If both $Y_{p c}=1$ and $x_{p k l}=1$, which means part $p$ is processed in cell $c$ and it requires machine $k$ immediately before $l$, machine $l$ should be placed after machine $k$ in cell $c$, not necessarily immediately. Constraints 6 and 7 guarantee that two machines cannot occupy the same place in a cell. Constraint 8 counts the number of skippings for each part, which is used as a surrogate objective to measure the material handling activities in a flow-line manufacturing cell.

\section{A numerical example}

The application of the proposed model to a small cell formation problem is now illustrated. Data for this example 
Table 3. Proposed part-family machine-cell formation.

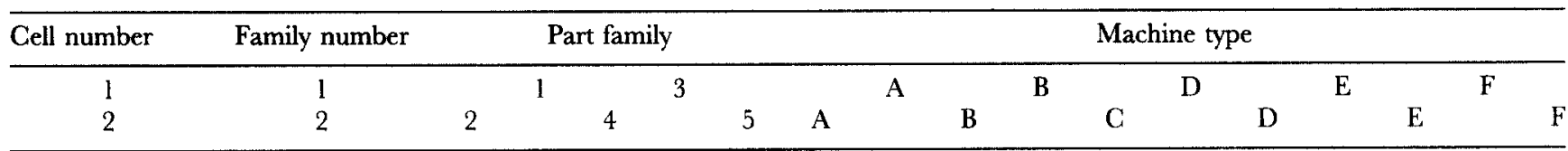

have been taken from Kamrani and Parsaei (1994), in which they considered five parts and six machine types. The operation sequences for each part are given in Table 1. The final solution of their methodology is summarized in Table 2. The flow patterns within each cell are shown in Figure 1, where the bold lines indicate the intercell moves and the numbers on each arrow is the part number.

As it can be seen from Figure 1, there are some intercell moves for all the parts, except part 1 . We can easily conjecture that a part-family machine-cell formation ignoring the within-cell layout problem might degrade the effectiveness of CM systems. Therefore, a new dissimilarity measure between parts $p$ and $r, O S_{p r}$, is proposed based on their operation sequences considering both the processing requirements and the flow direction in a cell to accomplish a modified flow-line structure. Tam (1990) represented an operation sequence by a string of characters, and used the Levenshtein distance measure between two strings to calculate the pairwise similarity matrix. We revised the Levenshtein distance measure, since the original measure does not penalize neither the backtracking parts nor the disjoint strings, as follows:

$z_{p r}= \begin{cases}H & \text { if two strings carry opposite sequences } \\ \frac{M N_{p r}}{\operatorname{COM}_{p r}} & \text { if } C O M_{p r}>0 \\ (h) \cdot M N T_{p r} & \text { if } C O M_{p r}=0\end{cases}$

where,

$z_{p r} \quad:$ dissimilarity value between strings $p$ and $r$

$M N T_{p r}$ : minimum number of insertions and deletions required to transform string $p$ to $r$

$C O M_{p r}$ : number of common operations in strings $p$ and $r$

$H \quad$ : a big constant number

$h \quad:$ a constant for penalizing disjoint strings, and $1<h \ll H$

The constant number $H$ is used to prevent two parts with backtracking sequences being assigned to the same cell by assigning a high dissimilarity value. If two parts have no common operations, then we find a dissimilarity value by using the penalizing factor $h$. Finally, $O S_{p r}$ matrix is found by applying an eigenvalue normalization on $z_{p r}$, if any entity of $z_{p r}$ is strictly greater than 1 . For the same example, $z$ and $O S$ matrices are given below.

$$
\begin{gathered}
z=\left[\begin{array}{l|lllll} 
& 1 & 2 & 3 & 4 & 5 \\
\hline 1 & 0 & & & & \\
2 & 4 & 0 & & & \\
3 & 0 \cdot 67 & 12 & 0 & & \\
4 & 1 & 1 \cdot 5 & 1 & 0 & \\
5 & 0 \cdot 5 & 2 & 0.5 & 0 \cdot 2 & 0
\end{array}\right] \\
O S=\left[\begin{array}{l|l|lllll}
1 & 2 & 3 & 4 & 5 \\
\hline 1 & 0 & 0 \cdot 3076 & 0 & & & \\
3 & 0 \cdot 0512 & 0 \cdot 9230 & 0 & & \\
4 & 0 \cdot 0769 & 0 \cdot 1154 & 0 \cdot 0769 & 0 & \\
5 & 0 \cdot 0384 & 0 \cdot 1538 & 0 \cdot 0384 & 0 \cdot 0154 & 0
\end{array}\right]
\end{gathered}
$$

In order to explain the proposed dissimilarity measure, we calculate the dissimilarity coefficient between parts 1 and 3 . The number of common operations, $\operatorname{COM}_{1,3}$, is equal to 3 , and the required number of insertions and deletions to transform the operation sequence of part 1 to part $3, M N T_{1,3}$, is 2. Therefore, $z_{1,3}$ is equal to $\frac{2}{3}$. After the eigenvalue normalization $O S_{1,3}=0 \cdot 0512$. On the other hand, the number of common operations between parts 2 and 3 is equal to zero, and $M N T_{2,3}=6$. Therefore $z_{2,3}=2 \times 6=12$, where $h$ is taken as 2 . The $k$-medoid technique is, then, applied on $O S$ for a given number of cells, $c=2$, to group parts into the families and to form machine cells. The objective is to minimize the average dissimilarity of each part to other parts in the same family. A more detailed discussion on the $k$-medoid technique can be found in Kaufman and Rousseeuw (1989). The final solution of the proposed approach is given in Table 3, while the flow patterns in each cell are shown in Figure 2. In the proposed cell layout, there are no intercell moves and only few skipping as compared to Figure 1. This illustrated that the proposed dissimilarity measure based on operation sequences is much stronger than any approach that uses only machining requirements as an input.

\section{Conclusions}

In this study, we propose a new approach that can be used to solve the within-cell layout problem in conjunction with the part family machine-cell formation problem. This paper also presents a similarity coefficient that takes into account the similarity of both machining requirements and the operation sequences of parts to establish a modified flow-line 


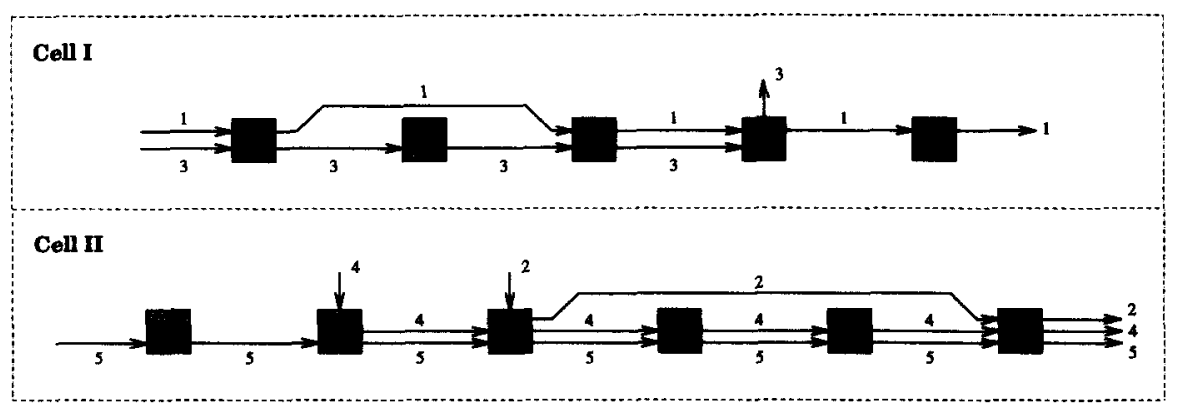

Figure 2. Proposed within-cell layout.

structure in each cell. An example problem studied in the previous section indicated that the proposed approach is more effective in terms of minimizing the material handling cost, streamlining the material flow in each cell and representing the basis for the design and implementation process of advanced manufacturing systems such as JIT and FMS. For future research, a new similarity measure can be developed, which can handle alternative process plans.

\section{References}

Choobineh, F., 1988, A framework for the design of cellular manufacturing systems. International Joumal of Production Research, 26, 1161-1172.

GreENE, T. J., and SADOWSKI, R. P., 1982, Cellular manufacturing control. Joumal of Manufacturing Systems, 2, 137-144.

Gunasekaran, A., Goyal, S. K., VirTanen, I., and Ylu-Olu, P., 1994, An investigation into the application of group technology in advanced manufacturing systems. International foumal of Computer Integrated Manufacturing, 7, 215-228.
Harhalakis, G., Nagi, R., and Proth, J. M., 1988, An efficient heuristic in manufacturing cell formation for group technology applications. International foumal of Production Research, 28, 185198.

KAMRANI, A. K., and PARSAeI, H. R., 1994, A methodology for the design of manufacturing systems using group technology. Production Planning \& Control, 5, 450-464.

KaufMan, L., and Rousseeuw, P. J., 1989, Finding Groups in Data (John Wiley \& Sons, New York).

Offodile, O. F., Mehrez, A., and Grznar, J., 1994, Cellular manufacturing: a taxonomic review framework. Joumal of Manufacturing Systems, 13, 196-220.

SARKeR, B. R., WilhelM, W. E., and HogG, G. L., 1994, Measures of backtracking and bi-directional flow in one dimensional machine location problems. Production Planning $\mathcal{E}^{2}$ Control, 5, 282-291.

TAM, K. Y., 1990, An operation sequence based similarity coefficient for part families formation. Foumal of Manufacturing Systems, 9, 55-68.

VAKHARIA, A., and Wemmerlov, U., 1990, Designing a cellular manufacturing system: a materials flow approach based on operations sequences. IIE Transactions, 22, 84-97. 